\title{
Importance of PET/CT Scan Use in Planning Radiation Therapy for Lymphoma
}

\author{
Mitric-Askovic Milana ${ }^{1 *}$, Erak Marko ${ }^{1,2}$, Latinovic Miroslav, Dugandzija \\ Tihomir $^{1,2}$
}

\begin{abstract}
Background: Radiation therapy is a key part of the combined modality treatment for Hodgkin's lymphoma (HL) and non-Hodgkin's lymphoma (NHL), which can achieve locoregional control of disease. The 3D-conformal radiation oncology can be extended-field (EFRT), involved-field (IFRT) and involved node (INRT). New techniques have resulted in a smaller radiation field and lower dose for critical organs such as lung heart and breast. Materials and Methods: In our research, we made a virtual simulation for one patient who was treated in four different radiotherapeutic techniques: mantle field (MFRT), EFRT, IFRT and INRT. After delineatiion we compared dose-volume histograms for each technique. The fusion of CT for planning radiotherapy with the initial PET/CT was made using Softver Xio 4.6 in the Focal program. The dose for all four techniques was 36Gy. Results: Our results support the use of PET/CT in radiation therapy planning. With IFRT and INRT, the burden on the organs at risk is less than with MFRT and EFRT. On the other hand, the dose distribution in the target volume is much better with the latter. Conclusions: The aim of modern radiotherapy of HL and NHL is to reduce the intensity of treatment and therefore PET/CT should be used to reduce and not increase the amount of tissue receiving radiation.
\end{abstract}

Keywords: Radiation therapy - PET/CT - Hodgkin's lymphoma - non-Hodgkin's lymphoma - radiotherapy planning

Asian Pac J Cancer Prev, 16 (5), 2051-2054

\section{Introduction}

Lymphomas are types of the malignant tumors who originate from immune system cells in various stages of differentiation. They cause several morphological, immunological and clinical situations. All lymphomas are divided into two types: Hodgkin's lymphoma (hereinafter: HL) and non-Hodgkin's lymphoma (hereinafter: NHL).

Radiation therapy is a key part of the combined modality treatment of lymphoma, which achieves locoregional control of the disease. Radiation is a local treatment, and knowledge of anatomy is paramount in designing radiation fields (Barrett et al., 2009; Specht et al., 2011; Phungrassmi et al., 2013). Because of the evolving technology, such as advances in computer science and radiologic imaging, a new era in radiation oncology brought the concept of three-dimensional treatment planning. In recent years, 18F-fluoro-deoxyD-glucose positron emission tomography (FDG-PET) has been incorporated in the management of malignant lymphomas. The FDG-PET provides valuable information in staging because it screening and evaluating the whole body for detecting hypermetabolic malignat lesions (Kang et al., 2014). It is necessary to emphasise that the PET/CT has important role in a staging HL and NHL especially diffuse B large cell type. Moreover it gives information regarding the evaluation of response to chemotherapy, planing of radiotherapy and also for restaging and followup after therapy. Therapeutic implications for patients with Hodgkin's lymphoma (HL) and non-Hodgkin's lymphoma (NHL) emphasize the importance of initial accurate disease staging. Other than being a valuable staging tool, it provides an accurate prognostic information for both HD and NHL, since a reduction of FDG uptake after initial chemotherapy is a highly predictive factor of favorable outcome, while residual uptake is indicative of unfavorable prognosis (Hasbek et al., 2014).

PET helps to distinguish complete disease remission from partial response (Eich et al., 2004; Ghalibafian et al., 2008) which can lead to a change in treatment plan. FDG-PET is very valuable for radiotherapy purpose. Field delineation in radiotherapy is the most important application of PET/CT scan in radiotherapy. By accurate assessment of initially involved anatomic sites and response to chemotherapy, radiation oncologists can adjust the prescribed dose, reduce the volume of irradiation, and thus spare the surrounding healthy tissues. FDG-PET has been reported as a useful tool for the early identification of patients who may benefit from salvage therapy (Arnold et al., 2009). 
Current data using advanced imaging techniques suggest that involved lymph node radiotherapy (INRT) may be as effective as IFRT, and would further reduce doses to normal tissues such as the lungs, heart and breasts. The characteristic of modern radiation therapy are smaller target volumes and lower radiation dose. Because tumor control rates are excellent with current approaches, there is an opportunity for the reduction of treatment intensity in an attempt to minimize late effects, especially in younger patients.

\section{Materials and Methods}

In this research, we made a virtual simulation for one patient who was treated in four different radiotherapeutic techniqeus: Mantle field (MFRT), extended-field (EFRT), involved-field (IFRT) and involved-node (INRT). After doing delineation and making plan we compared dose-volume histograms (DVH) for each technique. Patient had an initial PET/CT scan, which was done before start of chemotherapy. New software programs allow more precise imaging acquisition and fusion, and newer PET-CT-simulator integrated programs allow PET and computerized tomography (CT) or magnetic resonance (MRI) parallel volume contouring (Figure 1). In radiotherapy planning, localization of the tumor is essential and therefore it is critical to have high-accuracy PET-CT co-registration with radiotherapy planning CT.

The position of patient during PET/CT has to be same as treatment position. The fusion of CT for planning radiotherapy with the initial $\mathrm{PET/CT}$ was made using Softver Xio 4.6 in the Focal program. The dose was for all four techniqeus $36 \mathrm{~Gy}$. The Softver has two options for the fusion- automatic and manual.

\section{Results}

The results which are shown confirm that the dose distribution is much better in INRT and IFRT than in EF and MF (Table 1). According to these data the coverage of target volume (PTV) is significantly better in INRT and IFRT than in MF (Table1). Furthermore arrangement of the target volumes is homogeneous with better dose escalation.

\section{Table 1. Coverage Targets PTV}

\begin{tabular}{lcccc}
\hline dose (Gy) & plan MF & plan EF & plan IF & plan IN \\
\hline $\min (\mathrm{Gy})$ & 16.15 & 14.57 & 23.95 & 32.8 \\
$\max (\mathrm{Gy})$ & 41.69 & 39.55 & 39.21 & 37.5 \\
$\operatorname{maen}(\mathrm{Gy})$ & 34.6 & 36.01 & 36.33 & 36.25 \\
\hline
\end{tabular}
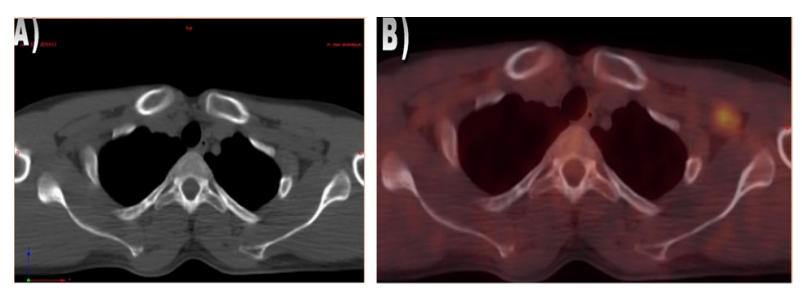

Figure 1. (A and B) IgI in the Left Axillary Area on CT and FDG-PET
Table 2. Spinal Cord

\begin{tabular}{lrcrc}
\hline dose (Gy) & plan MF & plan EF & plan IF & plan IN \\
\hline $\min (\mathrm{Gy})$ & 0.22 & 0 & 0.01 & 0 \\
$\max (\mathrm{Gy})$ & 34.25 & 37.6 & 8.64 & 0.11 \\
$\operatorname{maen}(\mathrm{Gy})$ & 1.80 & 14.17 & 2.38 & 0.02 \\
\hline
\end{tabular}

Table 3. Lung Left

\begin{tabular}{lcccc}
\hline dose (Gy) & plan MF & plan EF & plan IF & plan IN \\
\hline $\min (\mathrm{Gy})$ & 0.14 & 0.05 & 0.02 & 0 \\
$\max (\mathrm{Gy})$ & 39.47 & 38.38 & 38.1 & 35.31 \\
$\operatorname{maen}(\mathrm{Gy})$ & 13.24 & 6.91 & 5.61 & 1.23 \\
\hline
\end{tabular}

Table 4. Lung Right

\begin{tabular}{lcccc}
\hline dose (Gy) & plan MF & plan EF & plan IF & plan IN \\
\hline $\min (\mathrm{Gy})$ & 0.16 & 0.08 & 0 & 0 \\
$\max (\mathrm{Gy})$ & 39.3 & 37.6 & 4.59 & 0.05 \\
$\operatorname{maen}(\mathrm{Gy})$ & 10.5 & 6.91 & 0.21 & 0.01 \\
\hline
\end{tabular}

\section{Table 5. Heart}

\begin{tabular}{lcccc}
\hline dose (Gy) & plan MF & plan EF & plan IF & plan IN \\
\hline $\min (\mathrm{Gy})$ & 1.35 & 0.14 & 0.01 & 0 \\
$\max (\mathrm{Gy})$ & 39.62 & 32.58 & 25 & 0.18 \\
$\operatorname{maen}(\mathrm{Gy})$ & 18.58 & 2.6 & 0.24 & 0.02 \\
\hline
\end{tabular}

Table 6. Submandibular Left Gland

\begin{tabular}{lcccr}
\hline dose (Gy) & plan MF & plan EF & plan IF & plan IN \\
\hline $\min (\mathrm{Gy})$ & 33.19 & 30.9 & 0.1 & 0.01 \\
$\max (\mathrm{Gy})$ & 37.24 & 37.2 & 0.3 & 0.02 \\
$\operatorname{maen}(\mathrm{Gy})$ & 35.36 & 35.4 & 0.2 & 0.02 \\
\hline
\end{tabular}

On the other hand, the dose distribution on organ at risk is a lower in INRT and IFRT (Table 2,3,4,5,6,7,8). Minimum, maximum and mean dose on spinal cord, lung, heart and breast are significantly lower in INRT and IFRT. If the irradiated volume of an organ ar risk is smaller then the likelihood of complications in these organs is proprotionally lower.

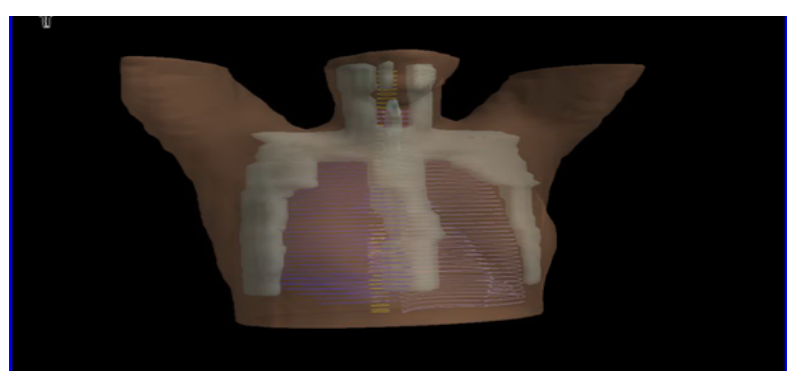

Figure 2. Mantle-field Radiotherapy (MFRT)

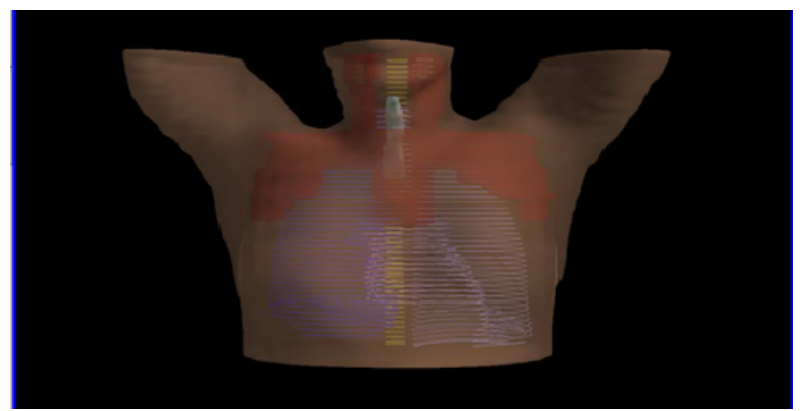

Figure 3. Extended Field Radiotherapy (EFRT) 
Table 7. Trachea

\begin{tabular}{lcrrc}
\hline dose (Gy) & plan MF & plan EF & plan IF & plan IN \\
\hline $\min (\mathrm{Gy})$ & 11.1 & 5.63 & 0.16 & 0 \\
$\max (\mathrm{Gy})$ & 36.3 & 37.94 & 1.36 & 0.05 \\
$\operatorname{maen}(\mathrm{Gy})$ & 30.6 & 28.71 & 0.51 & 0.01 \\
\hline
\end{tabular}

Table 8. Thyroid Gland

\begin{tabular}{lcccc}
\hline dose (Gy) & plan MF & plan EF & plan IF & plan IN \\
\hline $\min (\mathrm{Gy})$ & 15.2 & 6.48 & 0.1 & 0 \\
$\max (\mathrm{Gy})$ & 36.5 & 33.84 & 0.3 & 0.1 \\
$\operatorname{maen}(\mathrm{Gy})$ & 31.2 & 22 & 0.2 & 0 \\
\hline
\end{tabular}

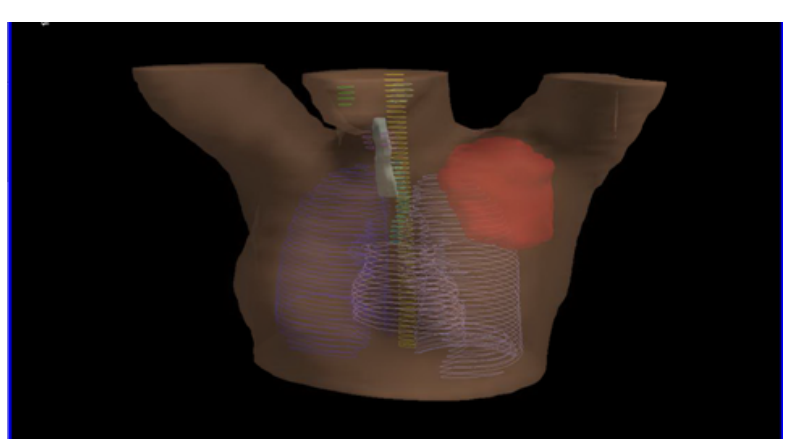

Figure 4. Involved-field Radiotherapy (IFRT)

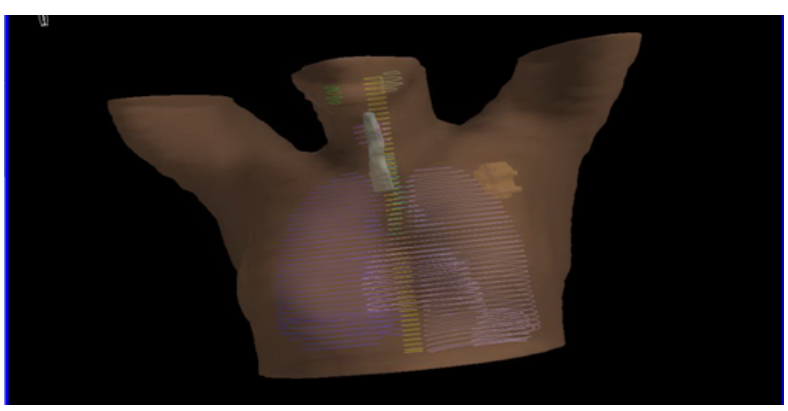

Figure 5. Involved-node Radiotherapy (INRT)

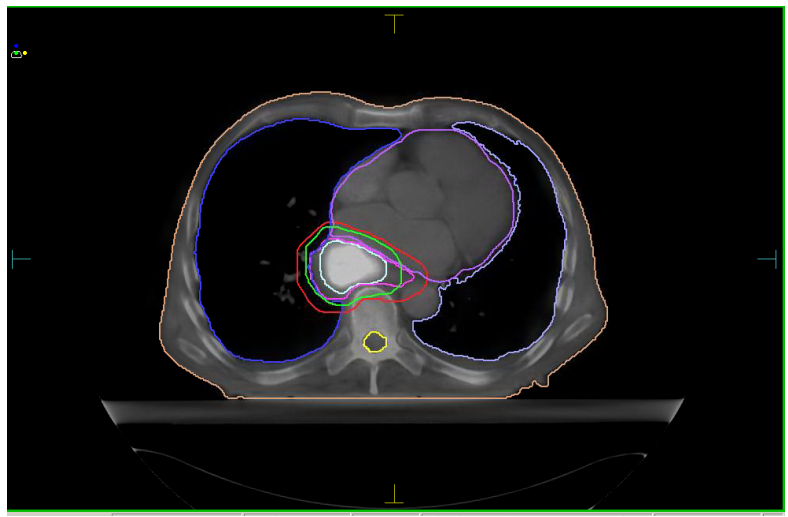

Figure 6. Delineation Based on Fusion with PET/CT

\section{Discussion}

Our results support the use of PET/CT in radiation therapy planning process, which can be seen from tables 1-8. In IFRT INRT (Figure 4,5) burden on the organs of risk is less than in the MF and $\mathrm{EF}$ (Figure 2,3; Table 1). On the other hand, the dose distribution in the target volume is much better. According to the data from PET/CT as one of advanced imaging technigues we know that involved lymph node radiotherapy (INRT) may be as effective as IFRT, and it would further reduce doses to normal tissues such as the lungs, heart and breasts.

The characteristic of modern radiation therapy are smaller target volumes and lower radiation dose.

PET can reveal targets that are not well visualised by $\mathrm{CT} /$ magnetic resonance (MR) structural imaging. Sometimes target can be distant from the unsuspected lymph node on CT, or there can be additional neoplastic masses adjacent to the target volume defined by CT or MR. Even more, some regions which are included in target volume on CT/MR do not actually contain tumor such as being reactive lymphadenopathy (IAEA, 2008).

The study by Hutchings and colleagues showed a high potential of PET to change the design of involved treatment fields in Hodgkin lymphoma (Lee et al., 2004). PET commonly influences RT fields in lymphoma by upstaging small nodes that are negative by structural imaging criteria or by demonstrating disease in sites where there is inadequate contrast between lymphoma and normal tissues on CT, such as spleen, liver, salivary glands and bowel (Figure 6). A case with early relapse of Hodgkin's lymphoma in an unirradiated CT-negative but 18F-FDG-positive lymph node region, published by the German Hodgkin's lymphoma study group, clearly showed the potential benefit of the consideration of 18F-FDG findings for RT planning (IAEA, 2004). The review article by Kostakoglu and Goldsmith showed that specificity values of FDG-PET for lymphoma exceed that of CT, which suggests that the GTV, which is delineated only based on information from CT scan, is showing fibrosis or enlarged, non-malignant nodes. Cremerius et al. had shown five negative-PET in HD and NHL stage II disease that were confirmed true by biopsy or follow-up data. In all cases, prescription doses of 30-40 Gy were used. Doses of 35 Gy to the lungs and breast region have been associated with a significant increase in the risk of secondary malignancies. Therefore, unnecessary treatment of normal tissue should be minimized (Table 2-8) (Lee et al., 2004). The improved staging provided by FDG-PET/CT should be used to decrease rather than increase the treated volumes (Hutchings et al., 2004). The EORTC group decided to develop a simple and practical methodology for INRT, and they advise that all the patients should have a CT and FDG-PET examination in the treatment position prior to chemotherapy, and a CT simulation after chemotherapy (Girinskya et al., 2008). PET can be useful for the evaluation of residual masses after chemotherapy in lymphoma, thus helping to determine which regions, if any, require radiation therapy and aiding to choose between a lower dose for presumed microscopic residual disease or a higher dose for gross residual disease (IAEA, 2008).

In conclusion, according to our results the use of PET/ $\mathrm{CT}$ in radiotherapy planning leads to better dose escalation of target volumes and control of organs at risk. The possibility of complications are lower which improve the quality of radiotherapy. The data from literatures suggest that the aim of modern radiotherapy HL and NHL is to reduce the intensity of treatment and therefore PET/CT should be used to reduce and not increase the amount of tissue receiving radiation. Today, with modern techniques it is possible to customize radiotherapy for each patient 
with the accurate delivery of radiation to the initially involved volume while minimizing the radiation dose to normal tissues. Our results also confirm this statement.

\section{References}

Arnold CP, Bin S (2008). The PET-CT in Radiotherapy Treatment Planning. 1st ed. Philadelphia: Saunders, An Imprint of Elsevier.

Barret A, Dobbs J, Morris S, Roques T (2009). Practical Radiotherapy Planning. 4th ed. London. Hodder Arnold.

Beyzadeoglu M, Ozyigit G, Ebrul C (2010). Basic Radiation Oncology; Springer-Verlag Berlin Heidelberg.

Eich HT, Staar S, Gossmann A, et al (2004). Centralized radiation oncologic review of cross-sectional imaging of Hodgkin's disease leads to significant changes in required involved field-results of a quality assurance program of the German Hodgkin Study Group. Int J Radiat Oncol Biol Phys, 58, 1121-7.

Engert A, Schiller P, Josting A, et al (2003). Involved-field radiotherapy is equally effective and less toxic compared with extended-field radiotherapy after four cycles of chemotherapy in patients with early-stage unfavourable Hodgkin's lymphoma: results of the HD8 trial of the German Hodgkin's Lymphoma Study Group. J Clin Oncol, 21, 3601-8.

Girinskya T, Ghalibafian M, Bonniaud G, Bayla A, Magne N, et al. (2007).Is FDG-PET scan in patients with early stage Hodgkin lymphom aof any value in the implementation of the involved-node radiotherapy concept and dose painting? Radiotherapy Oncology, 85, 178-86.

Girinskya T, Specht L, Ghalibafian M, et al (2008). The conundrum of hodgkin lymphoma nodes: To be or not to be included in the involved node radiation fields. The EORTC-GELA lymphoma group guidelines. Radiotherapy Oncol, 88, 202-21.

Girinskya T, Maazenb R, Spechtc L, et al (2006). Involvednode radiotherapy (INRT) in patients with early Hodgkin lymphoma: Concepts and guidelines. Radiotherapy Oncol, 79, 270-7.

Ghalibafian M, Beaudre A, Girinsky T (2008). Heart and coronary artery protection in patients with mediastinal Hodgkin lymphoma treated with intensity-modulated radiotherapy: dose constraints to virtual volumes or to organs at risk? Radiother Oncol, 87, 82-8.

Haioun C, Itti E, Rahmouni A, et al (2005).[18F]Fluoro-deoxyDglucose positron emission tomography (FDG-FDG-PET) in aggressive lymphoma: an early prognostic tool for predicting patient outcome. Blood, 106, 1376-81.

Hasbek Z, Yucel B, Salk I, et al (2014). Potential impact of atelectasis and primary tumor glycolysis on F-18 FDG PET/ $\mathrm{CT}$ on survival in lung cancer patients. Asian Pac J Cancer Prev, 15, 4085-9.

Hutchings M, Loft A, Hansen M, Berthelsen AK, Specht L (2007). Clinical impact of FDG-PET/CT in the planning of radiotherapy for early-stage Hodgkin lymphoma. Eur $J$ Haematol, 78, 206-12.

Hutchings M, Eigvea I, Specht L (2004). FDG-PET in the clinical management of Hodgkin lymphoma. Crit Rev Oncol Hematol, 52, 19-32.

IAEA (2008). The role of PET/CT in radiation treatment planning for cancer pantient treatment. Printed by the IAEA in Austria.

Kang P, Seo W, Lee S, et al (2014). Incidental abnormal FDG uptake in the prostate on 18-fluoro-2-Deoxyglucose PET-CT scans. Asian Pac J Cancer Prev, 15, 8699-703.

Kheng-Wei N, George M (2011). Role of radiotherapy in modern treatment of Hodgkin's lymphoma. Hindawi
Publishing Corporation Advance in Hematology, publishing Corporation Advance in Hematology.

Kostakoglu L, Coleman M, Leonard JP, et al (2002). PET predicts prognosis after 1 cycle of chemotherapy in aggressive lymphoma and Hodgkin's disease. J Nucl Med,43, 1018-27.

Lee YK, Cook G, Flower MA, et al (2004). Addition of 18F-FDG-FDGPET scans to radiotherapy planning of thoracic lymphoma. Radiother Oncol, 73, 277-83.

Mikhaeel NG, Hutchings M, Fields PA, et al (2005). FDGPET after two to three cycles of chemotherapy predicts progression-free and overall survival in high-grade nonHodgkin lymphoma. Ann Oncol, 16, 1514-23.

NCCN Clinical Practice Guidelines in Oncology V.2.2010. www.ncen.org.

Nieder C, Schill S, Kneschaurek P, Molls M (2007). Comparison of three different mediastinal radiotherapy techniques in female patients: impact on heart sparing and dose to the breasts. Radiother Oncol, 82, 301-7.

Paulino AC, Margolin J, Dreyer Z, Bin S, Teh SC (2001). Impact of PET-CT on involved field radiotherapy design for pediatric Hodgkin lymphoma. Pediatric Blood \& Cancer, 58, 860-4.

Phungrassami T, Funsian A, Spirlung H, (2013). 30 Years of radiotherapy service in Southern Thailand: workload vs resources. Asian Pac J Cancer Prev, 14, 7743-8.

Schinagl DA, Hoffmann AL, Voge VW, et al (2009). Can FDG-PET assist in radiotherapy target volume definition of metastatic lymph nodes in head and nech cancer? Radiother Oncol, 91, 95-100.

Spaepenk K, Stroobants S, Verhoef G, et al (2003). Positron emission tomography with [(18)F]FDG for therapy response monitoring in lymphoma patients. Eur J Nucl Med Mol Imaging, 30, 97-105.

Specht L, Yahalom J (2011). Radiotherapy for Hodgkin Lymphoma. Springer-Verlag Berlin Heidelberg. 